\title{
Photonic Band Characteristics in a Metallic Waveguide
}

\author{
Laxmi Shiveshwari and Biswadeep Chakraborty
}

\begin{abstract}
We study the transmission coefficient and photonic band characteristics in an inhomogeneous metallic circular waveguide with perfect conducting walls and having periodic variation of dielectric constant along the axial direction by the transfer matrix method. The propagation ceases below the waveguide cutoff and above that band characteristics are found to set in for different waveguide modes. In order to avoid multiple waveguide mode scattering by the superlattice, the dimension of the guide is chosen in such a manner that at the operating frequency, only the lowest mode can occur. We investigate the number of unit cells $(\mathrm{N})$ dependent transmission coefficient of the structure and also when acting as single or double electromagnetic barrier. Specifically, we demonstrate the existence of super narrow transmission band and a gap region for experimentally realizable structures. Photonic crystals have important applications in optical filtering with super narrow transmission band. For the guiding structure of infinite transverse extent, the analysis is found to agree well with that of wave propagation normal to the superlattice planes.
\end{abstract}

Index Terms-Photonic crystals, photonic band gap, transmission coefficient, dispersion relation.

\section{INTRODUCTION}

Waveguides [1], being the most basic structures in integrated optics, are extensively used for transmission of microwave power. It exhibits cutoff characteristic frequency similar to that of high pass filter. Due to translational invariance of the guiding structure, it is useful to single out the spatial variation of the fields in the z-direction and to assume $\vec{H}(x, y, z)=\vec{H}(x, y) e^{ \pm i \gamma z}$, where $\gamma$ is the propagation constant along $\mathrm{z}$ direction. If periodic perturbation is introduced, the guiding structure [2,3] can give spectrum with forbidden and allowed frequency band called photonic band structure (PBS)[4] which may be used for influencing the spontaneous emission (SE) [5] in addition to transport of energy or information. The active medium, for existence of PBS and observation of inhibition of SE, may have 3D periodic structure. The band gap properties of conventional photonic crystals (PCs) have been extensively studied [4]. Recent theoretical and experimental works suggest that 1D PCs [6-9] are an attractive candidate for this purpose. The 1D PCs are finite in space so they can not be studied as an infinite structure. From a theoretical point finite periodic systems are more difficult to analyze because

Manuscript received March 6, 2012; revised April 23, 2012.

Laxmi Shiveshwari is with the Department of Physics, K. B. Womens's College, Hazaribag 825 301, India (e-mail: hnsinha2003@gmail.com, Tel: +916546 272665, fax: +916546 270104).

Biswadeep Chakraborty is with the Department of Physics, Vinoba Bhave University, Hazaribag 825 301, India.
Bloch's theorem which dramatically simplifies the periodic problem does not apply. The finitely periodic case can be solved analytically for arbitrary $\mathrm{N}$ using the transfer matrix approach. This paper is concerned with a theory of PBS in an inhomogeneous metallic waveguide with perfect conducting walls and having periodic variation of dielectric constant along the axial direction only. In fact, the behaviour of the electromagnetic field in the optical waveguide with a stratified medium inside is analyzed. The 1D stratified medium of infinite transverse extent has been studied extensively. Here, we consider 1D finite $\mathrm{PC}$ which is of finite transverse extent. We develop the general theory for the guiding structure. The formulation of the problem is done so that we get scalar wave equation for the longitudinal component of the electric and magnetic fields. The component $\mathrm{H}_{\mathrm{z}}$ satisfies the Helmholtz equation whose solution has been obtained through the transfer matrix formulation.

\section{GENERAL THEORY}

Monochromatic EM wave of frequency $\omega$ in an inhomogeneous, isotropic dielectric medium is described by the vector wave equation as

$\nabla^{2} \vec{H}+(\vec{\nabla} \ln \varepsilon) \times(\vec{\nabla} \times \vec{H})+\omega^{2} \mu \varepsilon \vec{H}+\vec{\nabla}(\vec{H} \cdot \vec{\nabla} \ln \mu)=0$

We have $\mu=\mu_{0}$ for non magnetic materials and $\varepsilon=\varepsilon(z)$ for inhomogeneity along axial direction. Eq. (1)

reduces to $\quad \nabla^{2} H_{z}+\mu_{0} \omega^{2} \varepsilon(z) H_{z}=0$.

Solution of this equation is obtained as

$H_{z}=\sum_{m=0}^{\infty} \sum_{n=1}^{\infty} H_{0} Z(z) J_{m}\left(\gamma_{m n} \rho\right)\left(A_{m n} \cos m \phi+B_{m n} \sin m \phi\right)$,

by separation of variables in cylindrical co-ordinates, which is accomplished by the substitution

$$
H_{z}(\rho, \phi, z)=R(\rho) \Phi(\phi) Z(z) .
$$

Here, $\mathrm{H}_{0}, \mathrm{~A}_{\mathrm{mn}}$ and $\mathrm{B}_{\mathrm{mn}}$ are constants and $\mathrm{Z}(\mathrm{z})$ satisfies

$$
\frac{\partial^{2} Z}{\partial z^{2}}+\left(\mu_{0} \omega^{2} \varepsilon(z)-\gamma^{2}\right) Z=0 \text {. }
$$

Eq. (3) satisfies the requirements of finiteness at the origin. If there is centre conductor (co-axial line), the Neumann function cannot be excluded and in general must be retained to satisfy the boundary conditions at both inner and outer surfaces. The boundary conditions applicable here are: continuity of field components and Neumann boundary conditions. The surface boundary condition on $\mathrm{H}_{\mathrm{z}}$ gives 
$\frac{\partial H_{z}}{\partial \rho}=0$ at $\rho=R$, so that $J_{m}^{\prime}\left(\gamma_{m n} \rho\right)=0$ at $\rho=R$ which implies

$$
\gamma_{m n}=\frac{x_{m n}^{\prime}}{R}, \mathrm{n}=1,2,3,-----
$$

where $x_{m n}^{\prime}$ is the $\mathrm{n}^{\text {th }}$ root of the Eq. $J_{m}^{\prime}(x)=0$ and $\mathrm{R}$ is the radius of the metallic cylinder.

$\omega_{\lambda}=\frac{\gamma}{\sqrt{\mu_{0} \varepsilon}}$ is the cutoff frequency for the hollow metallic waveguide. For $\omega>\omega_{\lambda}$, the wave number is real, waves of different modes can propagate in the guide. We introduce periodicity into a hollow metallic circular waveguide assuming a Kronig slab dielectric consisting of alternate layers of dielectric materials $\mathrm{A}$ and $\mathrm{B}$ having dielectric constants $\varepsilon_{a}$ and $\varepsilon_{b}$ thicknesses a and b, respectively (Fig. 1),

i.e., $\quad \varepsilon(z)=\varepsilon_{b}+\left(\varepsilon_{a}-\varepsilon_{b}\right) \sum_{N} \theta[z-(N d+b)]$ and $\theta(z)=\left\{\begin{array}{l}1, z>0 \\ 0, z<0\end{array} . \mathrm{d}=\mathrm{a}+\mathrm{b}\right.$ is the width of the unit cell.

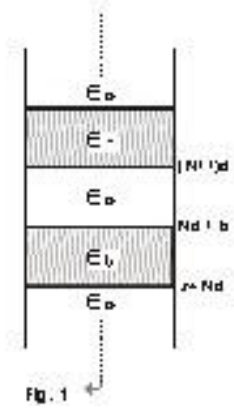

Fig. 1. Inhomogeneous metallic waveguide.

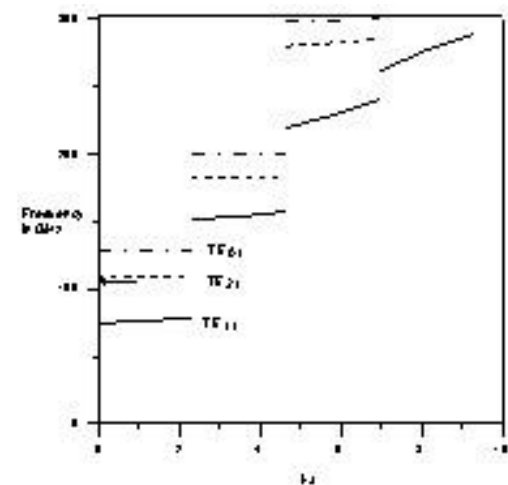

Fig. 2. Dispersion relation for a multilayer structure.

Assuming that modal fields are described by oscillatory nature in higher dielectric constant region and evanescent nature in lower dielectric constant region, $H_{z}(\rho, \varphi, z)$ for the $\mathrm{N}^{\text {th }}$ unit cell (inside the waveguide) can be written as

$H_{z}=H_{0} J_{m}(\gamma \rho)\left[A_{m n} \cos m \varphi+B_{m n} \sin m \varphi\right]\left[B_{N} e^{i k_{b}\{z-(N d+b)\}}+\bar{B}_{N} e^{-i k_{b}\{z-(N d+b)\}}\right]$, for $\mathrm{Nd}<\mathrm{z}<\mathrm{Nd}+\mathrm{b}$.

$$
H_{z}=H_{0} J_{m}(\gamma \rho)\left[A_{m n} \cos m \varphi+B_{m n} \sin m \varphi\right]\left[A_{N} e^{k_{a}\{z-(N+1) d\}}+\bar{A}_{N} e^{-k_{a}\{z-(N+1) d\}}\right] \text {,for } \mathrm{Nd}+\mathrm{b}<\mathrm{z}<(\mathrm{N}+1) \mathrm{d} \text {. }
$$

$k_{a}=\sqrt{\left(\gamma^{2}-\mu_{0} \omega^{2} \varepsilon_{a}\right)}$ and $k_{b}=\sqrt{\left(\mu_{0} \omega^{2} \varepsilon_{b}-\gamma^{2}\right)}$.

For TM modes, the basic solution $E_{z}=R(\rho) \Phi(\phi) Z(z)$ still applies, but the boundary condition on $\mathrm{E}_{\mathrm{z}}$ makes $\gamma_{m n}=\frac{x_{m n}}{R}$, where $\mathrm{x}_{\mathrm{mn}}$ is the $\mathrm{n}^{\text {th }}$ root of the Eq. $J_{m}(x)=0$. Continuity conditions at $\mathrm{z}=\mathrm{Nd}+\mathrm{b}$ and $\mathrm{z}=(\mathrm{N}+1) \mathrm{d}$ give the matrix equation

$$
\left(\begin{array}{l}
B_{N+1} \\
\bar{B}_{N+1}
\end{array}\right)=M\left(\begin{array}{l}
B_{N} \\
\bar{B}_{N}
\end{array}\right)
$$

where

$$
\begin{aligned}
& M_{11}=e^{i k_{b} b}\left[\cosh k_{a} a-\frac{i}{2}\left(\eta-\frac{1}{\eta}\right) \sinh k_{a} a\right]=M_{22}^{*} \\
& M_{12}=e^{i k_{b} b}\left[-\frac{i}{2}\left(\eta+\frac{1}{\eta}\right) \sinh k_{a} a\right]=M_{21}^{*} \\
& \text { Here } \eta=\left\{\begin{array}{l}
\frac{k_{a}}{k_{b}} \text { forTE mode } \\
\frac{k_{a} \varepsilon_{b}}{k_{b} \varepsilon_{a}} \text { forTM mode }
\end{array}\right. \text {. }
\end{aligned}
$$

The time-reversal symmetry together with conservation of energy leads to a general expression for transmission coefficient (TC) through $\mathrm{N}$ unit Cells, $\mathrm{T}_{\mathrm{N}}$, valid for any arbitrary $1 \mathrm{D}$ periodic structure

$$
\begin{aligned}
& T_{N}=\left[1+\frac{\sin ^{2} N \phi}{\sin ^{2} \phi}\left(\frac{1}{T_{1}}-1\right)\right]^{-1} \\
& \text { where } \phi=\cos ^{-1}\left\{\frac{1}{2} \operatorname{Tr}(M)\right\}
\end{aligned}
$$

is the Bloch phase for a single period. If $\mathrm{k}$ is the Bloch wave vector for infinite periodic structure,

$k=\frac{1}{d} \cos ^{-1}\left\{\frac{1}{2} \operatorname{Tr}(M)\right\}$ gives the dispersion relation (13)

\section{RESUlts AND Discussion}

Below the cutoff frequency of the waveguide every wave is subject to attenuation. Introducing step index periodicity along the axial direction into a hollow metallic circular cylinder can bring opaque bands above the cutoff frequency. Hereafter, we show the results of numerical calculation for metallic circular waveguide having periodic variation along axial direction with perfect conducting walls using Eq. (13). 
We first show the dispersion relation of EM modes for for $\varepsilon_{a}=\varepsilon_{0}$ (air), $\varepsilon_{b}=12.25 \varepsilon_{0}\left(\mathrm{Al}_{2} \mathrm{O}_{3}\right), \mathrm{a}=0.945 \mathrm{~mm}, \mathrm{~b}=$ $0.326 \mathrm{~mm}$ and $\mathrm{R}=0.5 \mathrm{~mm}$ for different TE modes in Fig. 2 . We see that at any given frequency only a finite number of modes can propagate. We can conveniently choose the dimension of the guide, so that at the operating frequency only the lowest mode can occur. This is shown by horizontal arrow on the Fig. 2. In this manner, we can ignore the multiple waveguide mode scattering by the superlattice, since one waveguide mode can be scattering into other guided modes. In the present work, we stress on the lowest TE mode which has $\mathrm{m}=\mathrm{n}=1$ while the lowest TM mode has $\mathrm{m}=0$ and $n=1$.Here, all relations derived for the TE case also hold for the TM case. We note that since $\vec{E}$ and $\vec{B}$ satisfy different boundary conditions at the interface between two dielectric media, the numerical values of the transmission coefficients and the transfer matrix will be different in general. We see that as frequency increases, $k_{a}$ decreases, correspondingly the decay rate of the field within the layers of medium A decreases. Increasing the frequency further, the modal fields are characterized by oscillatory nature in both the layers implying that propagation is not prohibited within layer A. This means that for the lowest mode, in the range from 0-175.9 GHz the propagation is forbidden in the individual layers A, and in the entire composite multilayer from 77.2-150.9, 158.5-220.1 and 241.5-261.4 GHz. Interestingly, in the frequency range between 241.5 to 261.4 $\mathrm{GHz}$, although propagation in the individual layers is allowed it is prohibited in the entire composite structure. In the following, we search for resonant tunneling with $\mathrm{N}=1$ (one layer of medium A) in Fig. 3 and $N=2$ (two layers of medium A) in Fig. 4 taking the dimension of the two layers a and $\mathrm{b}$ to be $0.945 \mathrm{~mm}$ and $0.326 \mathrm{~mm}$ and $\mathrm{R}$ to be $0.5 \mathrm{~mm}$ and $10 \mathrm{~cm}$ (cylinder of very radius). For $\mathrm{R}=0.5 \mathrm{~mm}$, we identify only one resonant point for $\mathrm{N}=1$. If for the given structural parameters we wish to have only the lowest mode, the operating frequency would be $\sim$ from 74.3 to $108.7 \mathrm{GHz}$. We see that there is no resonance for the single barrier problem in this frequency region on the other hand one resonance is present in the double barrier case. EM wave propagation is prohibited in the individual layers of medium A, therefore, the high transmission we see in Fig. 4 suggests a tunneling type of effect. It can transmit a mechanically suitable narrow band of frequency of light. This may find application in monochromators and optical filters.

Keeping all the parameters same as in Fig. 4 except $\mathrm{R}=1 \mathrm{~mm}$ we study the $\mathrm{N}$ dependent transmission of the multilayer guiding system and show our results in Fig. 5. In Fig. 6, we change the layer thicknesses as $a=0.326 \mathrm{~mm}$ and $b=0.945 \mathrm{~mm}$ keeping $\mathrm{R}$ as $1 \mathrm{~mm}$. We see that $T_{N}$ is rapidly fluctuating function of the Bloch phase $\phi$, when it is real. There are $(\mathrm{N}-1)$ transparent points of $\mathrm{T}_{\mathrm{N}}$ in addition to $\mathrm{T}_{1}=1$. We infer from Eq. (11) that these type of resonances depend on many parameters such as size of the barriers, distance between them, values of the dielectric constants and number of unit cells We observe that band characteristics are eminent even in an $\mathrm{N}=2$ system. When TC drops to a negligible value, $\omega$ crosses a band region to a gap region. For $\mathrm{N} \geq 2$, there are regions of almost vanishing TC. The result may be of immense significance in the experimental and computational investigations. We find that the cutoff frequency continuously shifts to lower frequency for thicker samples, reducing the band gap size. For $\mathrm{R} \rightarrow \infty$, i. e., when there is no transverse surface boundary, the situation is similar to wave propagation normal to the periodic layers for an all dielectric photonic structure. There is no cutoff frequency and band characteristics exist [7]. Homogeneous metallic waveguide is obtained by contracting one of the layer thicknesses to zero. Band characteristics disappear and waves can propagate above the cutoff frequency.

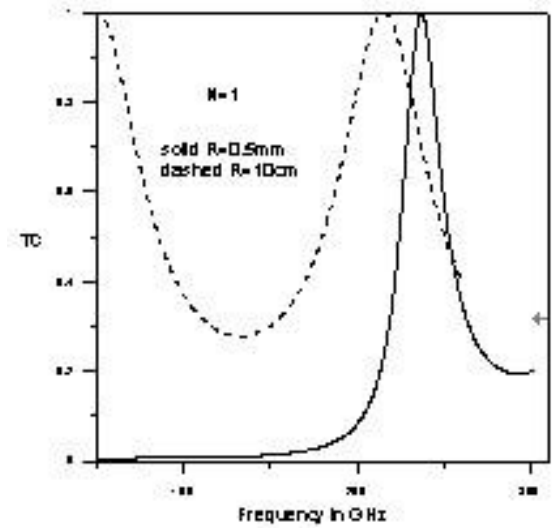

Fig. 3. Plot of TC versus frequency.

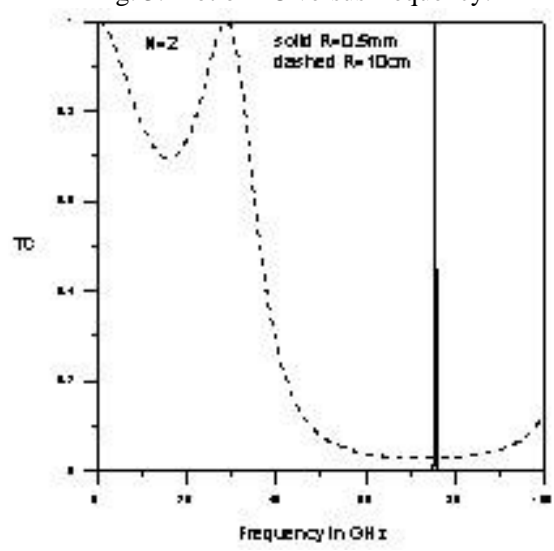

Fig. 4. Plot of TC versus frequency.

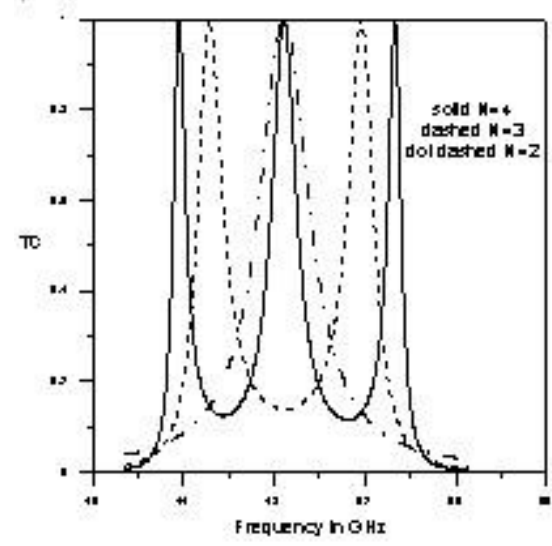

Fig. 5. Plot of TC versus frequency 


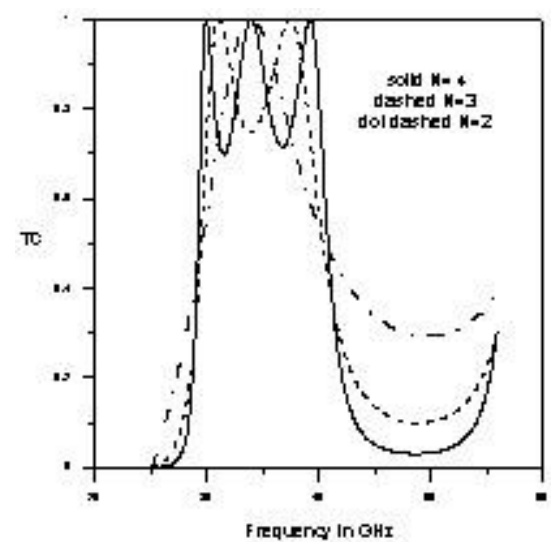

Fig. 6. Plot of TC versus frequency.

\section{SUMMARY}

We have theoretically analyzed the TC and PBG effect in a metallic waveguide filled with periodic dielectric materials. The central idea of this paper is that introducing periodicity into a hollow metallic circular waveguide can bring about new interesting optical properties. We have concentrated on the lowest mode only by choosing the appropriate dimension of the guide to neglect the complications arising due to multiple waveguide mode scattering by the superlattice. We have studied the multilayer structure that are experimentally resizable and can be fabricated more easily than 3D PBG systems by means of standard fabrication techniques which are routinely used in the optical industry. They may provide easy, cheap and effective alternative to improve the performance of various devices. The present! D PC can have important application in optical filtering with super narrow transmission band. When the circular waveguide is of infinite transverse extent, the situation is similar to the wave propagation in the stratification direction in the same PBG multilayer. But this will require more number of unit cells to have PBG characteristics. The most striking feature of the present study is the existence of super narrow transmission band and $\mathrm{PBG}$ characteristics in $\mathrm{N} \geq 2$ systems. Existence of cutoff frequency, formation of band characteristics and dependence of band gap on many parameters such as size of the barriers, distance between them, values of the dielectric constants etc. can be explained by the combined effect of waveguide cutoff attenuation and a global PBG effect. Multilayer guiding structure can offer enormous promise for optical communication, monochromators, optical filters and band gap engineering.

\section{ACKNOWLEDGEMENTS}

The authors are thankful to Dr. H. N. Sinha. The first author is thankful to UGC for providing financial support (MinorPproject No.PS-J-017/11-12 ERO).

\section{REFERENCES}

[1] J. D. Jackson, Classical Electrodynamics (John Wiley, New York, 1975).

[2] V. P. Bykov, Sov. J. Quantum Electron 4, vol. 861 (1975).

[3] R. A. Depine, M. L. M. Ricci, and A. Lakhtakia, "Microwave Opt. Technol," Lett. 48, 1945, 2006.

[4] J. D. Joannopoulos, R. D. Meade, and J. W. Winn, "Photonic Crystals: Molding the Flow of Light (Princeton University Press," Princeton, NJ, 1995.

[5] E. Yablonovitch, Phys. Rev. Lett. 58, 2059 (1989); S. John, Phys. Rev. Lett. 58, 2486, 1987.

[6] H. Daninthe, S. Foteinopoulou, and C.M. Soukoulis, "Photonics and Nanostructures-Fundamentals and applications," vol. 4, no. 123, 2006; Jon M. Bendickson and Jonathan P. Dowling and Michael Scalora, Phys. Rev. E 53, 4107 (1996).

[7] L. Shiveshwari and P. Mahto, "Solid State Commun," vol. 138, no. 160, 2006.

[8] L. Shiveshwari, Optik-Int. J. Light Electron Opt.122, 1523, 2011.

[9] W. Fan and L. Dong, Plasma Phys. vol. 17, no. 073506, 2010. 